\title{
Clinico-pathology, hematology and biochemistry responses in buffaloes towards Pasteurella multocida type B: 2 immunogen lypopolysaccharide via oral and intravenous routes of infection
}

\begin{abstract}
Haemorrhagic septicaemia is a disease caused by Pasteurella multocida serotype B: 2 and E: 2. The organism causes acute, highly fatal septicaemic disease with high morbidity and mortality in cattle and more susceptible in buffaloes. Lipopolysaccharide can be found on the outer cell wall of the organism. Lipopolysaccharide is released during multiplication which leads to inflammatory reaction. It represents the endotoxin of P. multocida type B: 2 and responsible for toxicity in haemorrhagic septicaemia which plays an important role in the pathogenesis of the disease. Therefore, the aim of this study was to investigate the clinical signs, blood parameters, gross post mortem lesions and histopathology changes caused by P. multocida type B:2 immunogen lipopolysaccharide infections initiated through intravenous and oral routes of infection. 9 buffalo heifers were divided equally into 3 treatment groups. Group 1 was inoculated orally with $10 \mathrm{ml}$ of phosphate buffer saline (PBS); Group 2 and 3 were inoculated with $10 \mathrm{ml}$ of lipopolysaccharide broth intravenously and orally respectively. For the clinical signs, there were significant differences $(\mathrm{p}<0.05)$ in temperature between the control, intravenous and oral group. In hematology and biochemistry findings, there were significant differences $(\mathrm{p}<0.05)$ in erythrocytes, haemoglobin, PCV, MCV, lymphocytes, monocytes, eosinophils, GGT and albumin between the control, intravenous and oral group. However, there were no significant differences $(p>0.05)$ in the MCHC, leukocytes, band neutrophils, basophils, thrombocytes, plasma protein, icterus index, total protein, globulin and A:G ratio between intravenous and oral group. For Group 2 buffaloes, there were gross lesions in the lung, trachea, heart, liver, spleen, and kidney. In contrast, lesions were only observed in the lung, trachea and liver of Group 3 buffaloes. There were significant differences $(\mathrm{p}<0.05)$ in hemorrhage and congestion; necrosis and degeneration; and inflammatory cells infiltration between experimental groups and control group. However, there were no significant differences $(p>0.05)$ in edema lesion between groups. In conclusion, this study is a proof that oral route infection of $\mathrm{P}$. multocida type B:2 immunogen lipopolysaccharide can be used to stimulate host cell responses where oral vaccine through feed could be developed in the near future.
\end{abstract}

Keyword: Buffalo heifers; Pasteurella multocida type B:2; Lipopolysaccharide; Intravenous inoculation; Oral inoculation; Clinico-pathology; Haematology; Biochemistry responses 